\title{
Chromosome Dynamics during Mitosis
}

\author{
Tatsuya Hirano \\ Chromosome Dynamics Laboratory, RIKEN, Wako, Saitama 351-0198, Japan \\ Correspondence: hiranot@riken.jp
}

The primary goal of mitosis is to partition duplicated chromosomes into daughter cells. Eukaryotic chromosomes are equipped with two distinct classes of intrinsic machineries, cohesin and condensins, that ensure their faithful segregation during mitosis. Cohesin holds sister chromatids together immediately after their synthesis during S phase until the establishment of bipolar attachments to the mitotic spindle in metaphase. Condensins, on the other hand, attempt to "resolve" sister chromatids by counteracting cohesin. The products of the balancing acts of cohesin and condensins are metaphase chromosomes, in which two rod-shaped chromatids are connected primarily at the centromere. In anaphase, this connection is released by the action of separase that proteolytically cleaves the remaining population of cohesin. Recent studies uncover how this series of events might be mechanistically coupled with each other and intricately regulated by a number of regulatory factors.

In eukaryotic cells, genomic DNA is packaged into chromatin and stored in the cell nucleus, in which essential chromosomal processes, including DNA replication and gene expression, take place (Fig. 1, interphase). At the onset of mitosis, the nuclear envelope breaks down and chromatin is progressively converted into a discrete set of rod-shaped structures known as metaphase chromosomes (Fig. 1, metaphase). In each chromosome, a pair of sister kinetochores assembles at its centromeric region, and their bioriented attachment to the mitotic spindle acts as a prerequisite for equal segregation of sister chromatids. The linkage between sister chromatids is dissolved at the onset of anaphase, allowing them to be pulled apart to opposite poles of the cell (Fig. 1, anaphase). At the end of mitosis, the nuclear envelope reassembles around two sets of segregated chromatids, leading to the production of genetically identical daughter cells (Fig. 1, telophase).

Although the centromere-kinetochore region plays a crucial role in the segregation process, sister chromatid arms also undergo dynamic structural changes to facilitate their own separation. Conceptually, such structural changes are an outcome of two balancing forces, namely, cohesive and resolving forces (Fig. 1, top left, inset). The cohesive force holds a pair of duplicated arms until proper timing of separation, otherwise daughter cells would receive too many or too few copies of chromosomes. The resolving force, on the other hand, counteracts the cohesive force, reorganizing each chromo-

Editors: Mitsuhiro Yanagida, Anthony A. Hyman, and Jonathon Pines

Additional Perspectives on Mitosis available at www.cshperspectives.org

Copyright (C) 2015 Cold Spring Harbor Laboratory Press; all rights reserved; doi: 10.1101/cshperspect.a015792

Cite this article as Cold Spring Harb Perspect Biol 2015;7:a015792 
T. Hirano
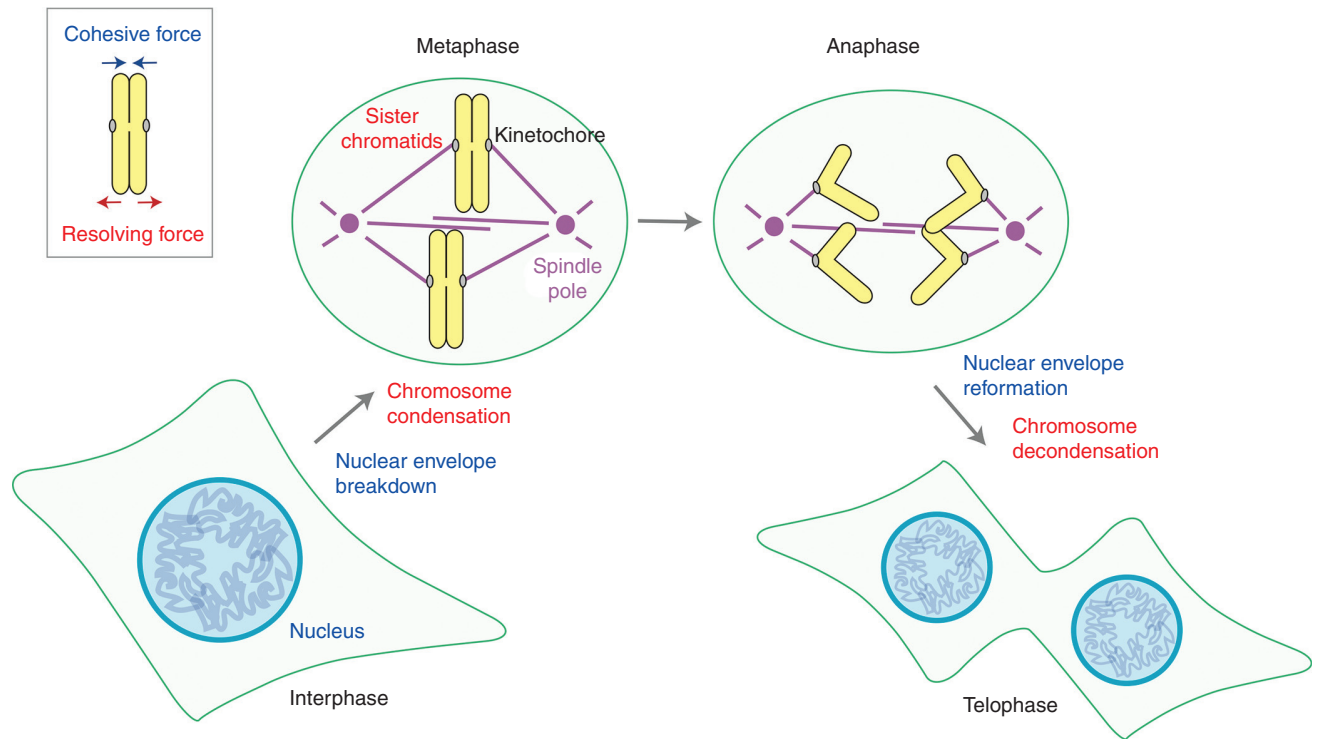

Figure 1. Overview of chromosome dynamics during mitosis. In addition to the crucial role of kinetochorespindle interactions, an intricate balance between cohesive and resolving forces acting on sister chromatid arms (top left, inset) underlies the process of chromosome segregation. See the text for major events in chromosome segregation.

some into a pair of rod-shaped chromatids. From this standpoint, the pathway of chromosome segregation is regarded as a dynamic process, in which the initially robust cohesive force is gradually weakened and eventually dominated by the resolving force. Almost two decades ago, genetic and biochemical studies for the behavior of mitotic chromosomes converged productively, culminating in the discovery of cohesin (Guacci et al. 1997; Michaelis et al. 1997; Losada et al. 1998) and condensin (Hirano et al. 1997; Sutani et al. 1999), which are responsible for the cohesive and resolving forces, respectively. The subsequent characterizations of these two protein complexes have not only transformed our molecular understanding of chromosome dynamics during mitosis and meiosis, but also provided far-reaching implications in genome stability, as well as unexpected links to human diseases. In this article, I summarize recent progress in our understanding of mitotic chromosome dynamics with a major focus on the regulatory networks surrounding cohesin and condensin. I also discuss emerging topics and attempt to clarify outstanding questions in the field.

\section{SUBUNIT ORGANIZATION OF COHESIN AND CONDENSINS}

Cohesin and condensin are two distinct classes of protein complexes, yet they are structurally related to each other (Fig. 2) (reviewed by Peters et al. 2008; Hirano 2012). The core of each complex is a heterodimeric pair of structural maintenance of chromosomes (SMC) ATPase subunits; cohesin contains a pair of SMC1 and SMC3, whereas condensin contains a pair of SMC2 and SMC4. The regulatory subunits of cohesin and condensin are also distantly related to each other. Cohesin contains a member of the kleisin family $(\operatorname{Rad} 21 / \mathrm{Scc} 1 / \mathrm{Mcd} 1)$ and a HEAT (Huntingtin, elongation factor 3 , A subunit of protein phosphatase 2A, TOR)-repeat-containing subunit (SA/Scc3). On the other hand, the canonical condensin complex (now known as condensin I) contains a kleisin subunit (CAP-H) and a pair of HEAT subunits (CAP-D2 and CAP-G). It has turned out that many, if not all, eukaryotic species have a second condensin complex (condensin II) that shares the same pair of SMC subunits with condensin I, but has a distinct set of regu- 


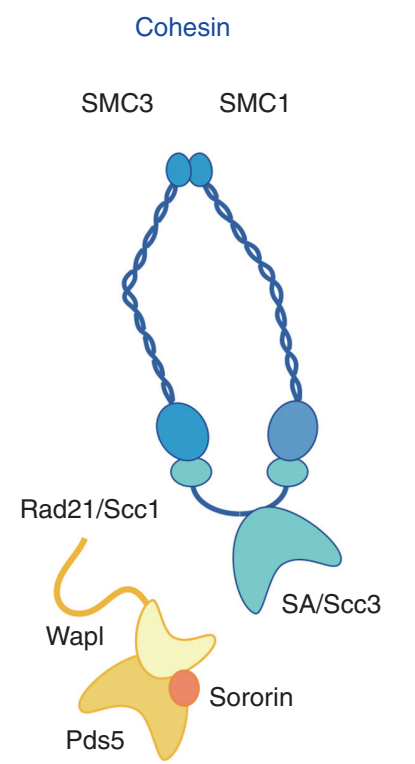

\author{
Condensin I
}

SMC2 SMC4

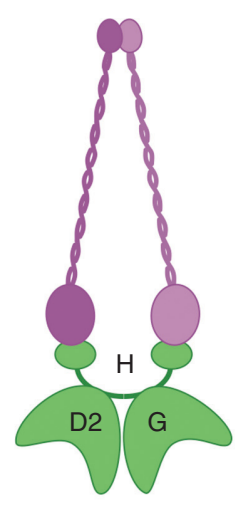

Condensin II

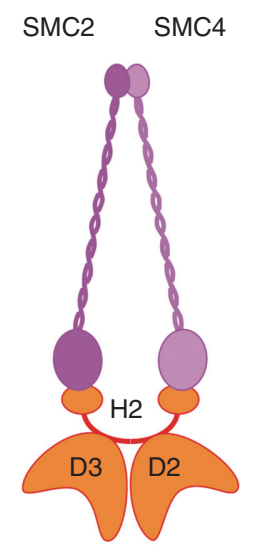

SMC core

subunits

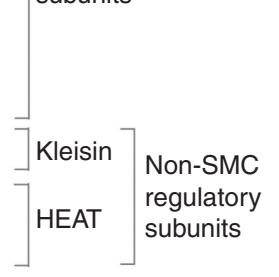

Figure 2. Subunit organization of cohesin and condensins. Cohesin is composed of a dimer of SMC1 and SMC3, a kleisin subunit ( $\operatorname{Rad} 21 / \mathrm{Scc} 1$ ), and a HEAT (Huntingtin, elongation factor 3, A subunit of protein phosphatase 2A, TOR)-repeat subunit (SA/Scc3). Condensin I and II share the same pair of SMC2 and SMC4, and contain distinct sets of non-SMC regulatory subunits; each set is composed of a single kleisin subunit (CAP-H for condensin I; CAP-H2 for condensin II) and a pair of HEAT-repeat subunits (CAP-D2 and CAP-G for condensin I; CAP-D3 and CAP-G2 for condensin II). The cohesin complex is further regulated by loosely associated factors, such as Wapl-Pds5 and sororin.

latory subunits, namely, a kleisin subunit (CAP$\mathrm{H} 2$ ) and a pair of HEAT subunits (CAP-D3 and CAP-G2). As discussed below, the cohesin complex has a number of additional regulatory factors, some of which loosely associate with the complex (e.g., Wapl-Pds5 and sororin).

\section{COHESIN ESTABLISHES SISTER CHROMATID COHESION DURING S PHASE}

Cohesin starts to associate with chromatin during $G_{1}$ phase and establishes sister chromatid cohesion during $S$ phase (Fig. $3 \mathrm{~A}, \mathrm{~B})$. It remains to be fully elucidated what really happens at this transition at a mechanistic level. Early studies had pointed out the involvement of a specialized class of acetyltransferases (Eco1/Ctf7 in budding yeast; $\mathrm{ESCO} 1$ and $\mathrm{ESCO} 2$ in vertebrates) (Ivanov et al. 2002; Bellows et al. 2003). A series of subsequent studies showed that two conserved residues in SMC3 are the essential targets of the Eco1/ESCO1 acetyltransferase, and the acetylation reactions are indeed essential for cohesion establishment in budding yeast (Ben-Shahar et al. 2008; Unal et al. 2008) and humans (Zhang et al. 2008). More recently, the deacetylase that reverses this reaction has been identified as Hos1 in budding yeast (Beckouët et al. 2010; Borges et al. 2010; Xiong et al. 2010) and HDAC8 in humans (Deardorff et al. 2012). It has been proposed that the deacetylation reaction plays an important role in "recycling" used cohesin complexes for the next cell cycle. It is also important to note that deficiencies in this acetylation/deacetylation cycle of cohesin cause developmental diseases in humans, such as Roberts syndrome (Vega et al. 2005) and Cornelia de Lange syndrome (Deardorff et al. 2012).

Cohesin acetylation and active DNA replication further recruit another protein called sororin to the cohesion sites (Lafont et al. 2010; Nishiyama et al. 2010). Sororin then displaces Wapl from its partner Pds5, thereby inhibiting Wapl's ability to help dissociate cohesin from 
T. Hirano

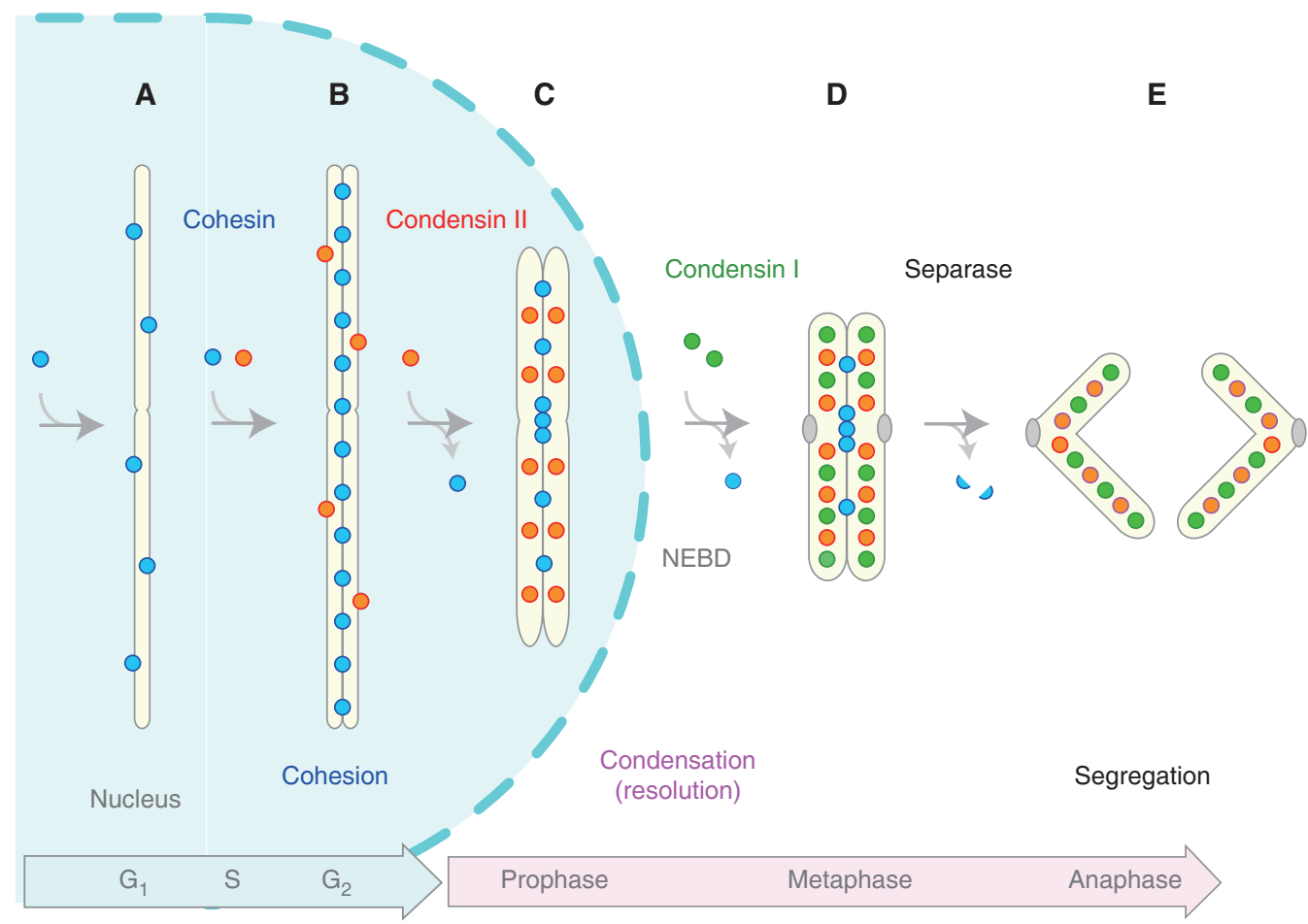

Figure 3. Overview of the behaviors of cohesin and condensins during the cell cycle (in vertebrate cells). $(A)$ Cohesin associates with chromatin in $\mathrm{G}_{1}$ phase, and $(B)$ establishes sister chromatid cohesion during $\mathrm{S}$ phase. A subfraction of condensin II starts to associate with duplicated regions of chromosomes during S phase and initiates sister chromatid resolution by counteracting cohesin. $(C)$ When cells enter mitotic prophase, most cohesin complexes are released from chromosome arms and more condensin II complexes are loaded, promoting an early stage of chromosome condensation within the nucleus. $(D)$ Upon nuclear envelope breakdown (NEBD) in prometaphase, the cytoplasmically located condensin I gains access to chromosomes and further facilitates chromosome condensation. ( $E$ ) At the onset of anaphase, separase cleaves the kleisin subunit of cohesin, thereby promoting irreversible separation of sister chromatids.

chromatin until early mitosis. Although Wapl and Pds5 are widely conserved from yeast to humans, the apparent orthologs of sororin have, so far, been found only among metazoans. Moreover, the cell-cycle stages at which the requirements for Wapl and Pds5 in regulating cohesin dynamics become most prominent differ among different species. It is nonetheless very clear that a large number of specialized factors form an intricate network so that cohesin's multipurpose actions are regulated very tightly and carefully throughout the cell cycle (also, see below).

Substantial lines of evidence have accumulated that the tripartite ring of cohesin (composed of SMC1-SMC3-Rad21/Scc1) encircles two sister DNAs to hold them together (re- viewed by Nasmyth 2011). The role of the fourth subunit of cohesin, stromal antigen (SA)/Scc3, is least understood. Moreover, vertebrate cells have three paralogs of SA (also known as STAG), which include two mitotic forms (SA1 and SA) and one meiotic form (SA3). An early study had shown that the relative ratio of SA1 to SA2 appears to differ among different cell types (Losada et al. 2000). Then many important questions arise. Why do vertebrate cells have two different mitotic paralogs? Do they have unique functions in mitotic chromosome dynamics? A recent study shed light on these questions by providing evidence that cohesin-SA1 and -SA2 are differentially required for telomere cohesion and centromere cohesion, respectively, in HeLa 
cells (Canudas and Smith 2009). Consistently, cells derived from SA1-null mice display defects in telomere cohesion, causing a high incidence of aneuploidy (Remeseiro et al. 2012a). Moreover, a genome-wide analysis of distribution of SA1 and SA2 revealed a unique role of cohesinSA1 in gene regulation, such as at the c-myc and protocadherin loci (Remeseiro et al. 2012b). It has also been reported that targeted inactivation of SA2 in a human cell line causes cohesion defects and aneuploidy (Solomon et al. 2011). In the future, it will be of importance to determine how cohesin-SA1 and -SA2 are targeted to specific loci at a mechanistic level, although there is evidence that SA1 might use its AT-hook motif for telomere binding (Bisht et al. 2013).

\section{CONDENSIN II INITIATES ITS ACTION LONG BEFORE ENTRY INTO MITOSIS}

Early studies showed that cell-cycle dynamics of condensins I and II are radically different from each other in HeLa cells (Hirota et al. 2004; Ono et al. 2004). For instance, condensin II is already nuclear during interphase, whereas condensin I is sequestered into the cytoplasm until the nuclear envelope breaks down at prometaphase. Then, what might condensin II do during interphase? A recent study has shown that condensin II initiates its action during $S$ phase and counteracts cohesin-mediated cohesion (Fig. 3B) (Ono et al. 2013). A combination of two functional assays, premature chromosome condensation (PCC) and fluorescence in situ hybridization (FISH), revealed that condensin II associates with duplicated regions of chromosomes and promotes their "resolution" during $S$ phase. In other words, contrary to popular views, sister chromatid cohesion is not a oneway process supported by cohesin; rather, the distance between sister chromatids is determined by the balancing acts of cohesin-mediated cohesion and condensin II-mediated resolution (Fig. 4A).

Although it remains unknown how condensin II might be targeted to duplicated regions of chromosomes during $\mathrm{S}$ phase, its negative regulator has been identified from an unexpected path of investigation. MCPH1 is a BRCA carboxy-terminal (BRCT) domain-containing
A

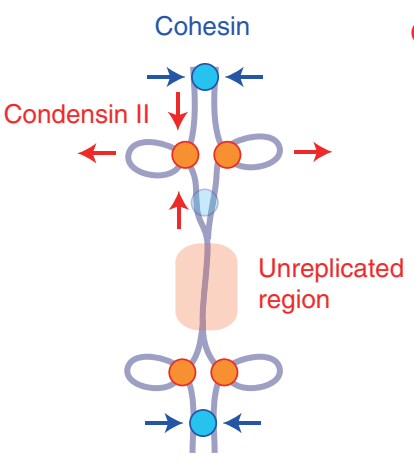

S phase
B

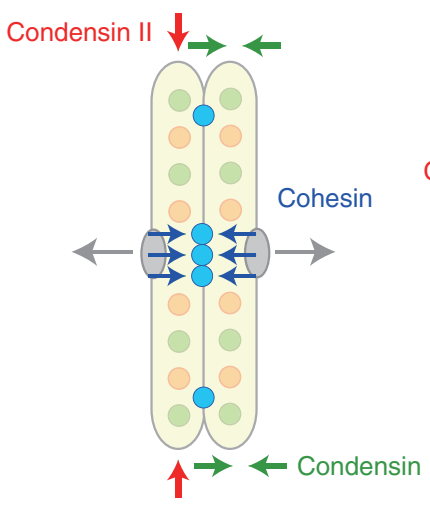

Metaphase
C

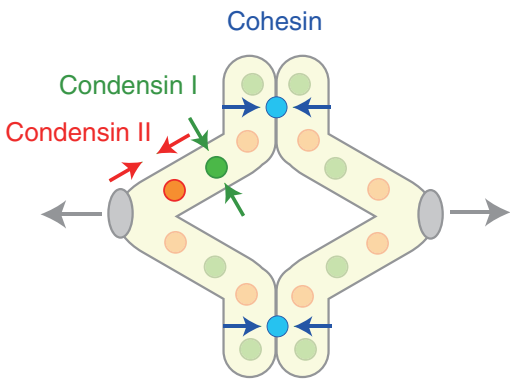

Anaphase

Figure 4. Balancing acts of cohesin and condensins control chromosome dynamics throughout the cell cycle. $(A)$ During $S$ phase, cohesin and condensin II counteract in newly duplicated regions of chromosomes. $(B)$ In metaphase, the coordinated acts of condensin I (lateral compaction) and condensin II (axial shortening) facilitate resolution and shaping of sister chromatid arms. Although cohesin is enriched at centromeres at this stage, a small fraction of cohesin is also left along arms and participates in arm cohesion. $(C)$ The balancing actions of cohesin and condensins persist until anaphase, thereby contributing to proper segregation of chromosome arms. 
protein whose mutations cause a neurodevelopmental disorder, known as primary microcephaly, in humans (reviewed by Thornton and Woods 2009). The original observation that MCPH1 patient cells display PCC in $\mathrm{G}_{2}$ phase led to the realization that MCPH1 could play a role in suppressing premature activation of condensin II during interphase (Trimborn et al. 2006). A more recent study using Xenopus egg extracts has provided evidence that human MCPH1 indeed acts as a highly specific and potent inhibitor of condensin II, but not of condensin I (Yamashita et al. 2011). MCPH1 is ubiquitously expressed, and MCPH1-knockout mice are viable, albeit displaying microcephaly (Gruber et al. 2011). Why MCPH1 mutations cause specific developmental defects in the brain, but not in other tissues, is an interesting topic for future studies.

A recent series of genetic studies in Drosophila melanogaster has shed additional and important insights into the role of condensin II subunits in regulating interphase chromatin architecture. In this organism, homologous chromosomes are paired in diploid somatic cells, leading to a specialized gene regulatory event, known as transvection, in which a gene is transcriptionally activated or repressed in trans by regulatory elements located on the homologous chromosomes. Remarkably, condensin II subunits have been shown to play a role in antagonizing transvection (Hartl et al. 2008), as well as somatic homolog pairing (Joyce et al. 2012). Moreover, condensin II subunits apparently contribute to chromosome territory formation in polyploid nurse cells (Bauer et al. 2012). Taken all together, it is reasonable to speculate that condensin II regulates not only the resolution of sister chromatids within individual chromosomes, but also the disruption of interchromosomal interactions within the interphase nucleus. Evidence has also been provided that such interphase functions of condensin II are down-regulated by the $\mathrm{SCF}^{\text {slimb }}$ ubiquitin ligase complex (Buster et al. 2013). It remains to be clarified, however, whether Drosophila indeed has a conventional type of condensin II because the Cap-G2 gene is apparently missing in its genome (Herzog et al. 2013).

\section{PROPHASE CONDENSATION MEDIATED BY CONDENSIN II COINCIDES WITH RELEASE OF COHESIN FROM CHROMOSOME ARMS}

Although condensin II participates in regulating interphase chromosome architecture, it is difficult to visualize such contribution by conventional staining with DNA-binding dyes under normal conditions in diploid cells. An early stage of chromosome condensation becomes discernible only after mitotic prophase (Kireeva et al. 2004) and this process, too, is mediated by condensin II (Fig. 3B,C) (Hirota et al. 2004; Ono et al. 2004). Recent studies have reported that this process depends on Cdk1- and Mps1mediated phosphorylation of the subunits of condensin II (Abe et al. 2011; Kagami et al. 2014). Mechanistically how condensin II might initiate prophase condensation remains unknown because no biochemical activities associated with condensin II have been reported to date. It is nonetheless tempting to speculate that the Cdk1-mediated phosphorylation stimulates a (putative) supercoiling activity of condensin II and thereby promotes structural reorganization of chromosomes, as had been shown for condensin I (Kimura et al. 1998; StPierre et al. 2009).

At the same time, when condensin II starts to act on prophase chromosomes, most cohesin is released from their arms, thereby weakening the physical linkage between sister chromatids (Fig. 3B,C). An outcome of the two temporally coordinated events is the appearance of individual chromosomes, in which a pair of sister chromatids becomes discernible from each other, a process known as sister chromatid resolution (reviewed by Shintomi and Hirano 2010). Early studies had suggested that direct phosphorylation of cohesin subunits by Plk1 might promote release of cohesin from chromosome arms (Losada et al. 2002; Sumara et al. 2002). However, this process turned out to be much more complex, requiring a specialized releasing factor, Wapl (Gandhi et al. 2006; Kueng et al. 2006), and its partner, Pds5 (Shintomi and Hirano 2009).

A pair of recent structural studies uncovered that the carboxy-terminal domain of Wapl is 
composed of HEAT repeats (Chatterjee et al. 2013; Ouyang et al. 2013), a repeat motif also found in Pds5. The interaction of Wapl-Pds5 with the cohesin complex is multilayered and highly complex: Wapl, for instance, interacts not only with its regulatory subunits (SA1$\operatorname{Rad} 21 /$ Scc1) (Gandhi et al. 2006; Shintomi and Hirano 2009), but also with the SMC3 head domain (Chatterjee et al. 2013). It will be of great interest and importance in the future to understand why HEAT repeats are found in many components of the cohesion and condensation machinery (Fig. 2), and how they might work at a mechanistic level.

\section{CONDENSIN I ASSOCIATES WITH CHROMOSOMES IN PROMETAPHASE AND SHAPES MITOTIC CHROMOSOMES}

As mentioned above, unlike condensin II, condensin I is sequestered into the cytoplasm during interphase and associates with chromosomes only after nuclear envelope breakdown (NEBD) at prometaphase (Fig. 3C,D). Such a difference in the subcellular localization of condensins I and II is widely observed among $\mathrm{Xe}$ nopus egg extracts (Shintomi and Hirano 2011), mouse oocytes (Lee et al. 2011), and even evolutionarily distant organisms, such as the primitive red alga Cyanidioschyzon merolae (Fujiwara et al. 2013), implicating its fundamental importance. Although it has been speculated that the differential regulation of the two condensin complexes could help specify their sequential actions (i.e., condensin II first, condensin I later), the physiological significance for such a mechanism remains to be fully understood. In this sense, it is important to note that, as judged by a fluorescence recovery after photobleaching (FRAP) analysis, condensin I interacts with metaphase chromosomes in a more dynamic manner than condensin II does (Gerlich et al. 2006a).

Then, how might the two condensin complexes coordinate the process of mitotic chromosome assembly? An early study showed that depletion of condensin I- or II-specific subunits in HeLa cells causes highly characteristic and distinct defects in metaphase chromosome architecture (Ono et al. 2003). A pair of recent studies has addressed the same question by using quantitative immunodepletion in Xenopus egg extracts (Shintomi and Hirano 2011) and conditional knockdown in chicken DT40 cells (Green et al. 2012). Data from the two studies uncovered, again, seemingly distinct functions of the two condensin complexes: Condensin II primarily contributes to axial shortening of chromosomes, whereas condensin I supports their lateral compaction (Fig. 4B). Thus, the relative ratio (and/or activity) of condensin I to II is likely to function as one of the key determinants in shaping metaphase chromosomes. This notion also provides a starting point for readdressing the long-standing question of how chromosome scaling and shaping might be regulated in a developmental stage-specific manner among different organisms.

From an evolutionary point of view, it is important to note that condensin II is absent in some species, including fungi (Hirano 2012). Moreover, the condensin II-specific subunits apparently play less-important roles than the condensin I-specific subunits during the mitotic chromosome cycle in organisms, such as Drosophila (Savvidou et al. 2005; Herzog et al. 2013), Arabidopsis thaliana (Sakamoto et al. 2011), and C. merolae (Fujiwara et al. 2013). Consistent with this view, genome-wide screens for genes affecting chromosome condensation in yeasts and Drosophila have identified condensin I and topoisomerase II (Hirano et al. 1986; Samejima et al. 1993; Goshima et al. 2007; Petrova et al. 2012), implicating that no more "major" condensation factors remain to be discovered, at least in these organisms. The situation could be different in organisms with larger genomes, however. For instance, it has been reported that KIF4, a chromokinesin, contributes to chromosome condensation and shaping in humans (Mazumdar et al. 2004) and chickens (Samejima et al. 2012). The mechanism by which this microtubule motor protein might regulate chromosome architecture remains unknown.

The molecular picture of how the localization and activities of condensin I might be regulated is far from complete, although numerous 
T. Hirano

studies have identified involvement of multiple mitotic kinases, including Cdk1 (Kimura et al. 1998; St-Pierre et al. 2009), Aurora B (Lipp et al. 2007; Collette et al. 2011; Nakazawa et al. 2011; Tada et al. 2011), and Polo-like kinase (St-Pierre et al. 2009). Sumoylation has also been implicated in the function of condensin in ribosomal DNA (rDNA) maintenance (Takahashi et al. 2008) and kinetochore localization (BachellierBassi et al. 2008) in budding yeast. It is noteworthy that, unlike the case of cohesin, no specialized loading or dissociating factors have, so far, been identified for condensins. One potential explanation for this might be that cohesin's association with chromatin is far less dynamic than condensin's (Gerlich et al. 2006a,b), and even a subtle change in cohesin's dosage has a big impact on its chromosomal functions (Gause et al. 2010; Heidinger-Pauli et al. 2010). Thus, it is most likely that cohesin demands a much tighter level of regulation compared with condensins.

\section{SEPARATION AND DYNAMICS OF SISTER CHROMATIDS IN ANAPHASE}

As mentioned above, although the coordinated action of Wapl-Pds5 and mitotic kinases, such as Plk1 and Aurora B, promotes release of bulk cohesin from chromosome arms, a residual level of cohesin is protected from this reaction by Sgo1-PP2A, remaining bound primarily at centromeres (Kitajima et al. 2006). At the onset of anaphase, this subpopulation of cohesin is proteolytically cleaved by the action of separase (Uhlmann et al. 1999), leading to the final and irreversible separation of sister chromatids (Fig. $3 \mathrm{D}, \mathrm{E})$. The protease activity of separase is negatively regulated by a protein known as securin, which itself is the target of the anaphase-promoting complex/cyclosome (APC/C). A recent work has shown that an autocleaved form of separase directly inhibits Cdk1 activity, thereby coordinating cohesin cleavage and cell-cycle progression (Shindo et al. 2012).

How do chromosome arms behave after the onset of anaphase when they are being transported into opposite poles? A series of recent studies has started to shed new light on this hitherto underappreciated question. For instance, a small yet substantial amount of cohesin remains to hold sister chromatid arms even after anaphase onset, thereby contributing to the coordination of their gradual separation and spindle elongation (Fig. 4C). It is most likely that Sgo1 plays a role in retaining this population of cohesin along arms, which is eventually cleaved by separase (Nakajima et al. 2007; Harrison et al. 2009). Likewise, strong lines of evidence indicate that the final separation of sister arms requires continuous actions of condensin and topoisomerase II throughout anaphase (Renshaw et al. 2010; Nakazawa et al. 2011; Tada et al. 2011). Comparison of biophysical properties between metaphase and anaphase chromatids by mechanical stretching experiments (Marko 2008) might be an interesting direction in the future.

\section{RESOLVING AND SEGREGATING DIFFICULT REGIONS OF CHROMOSOMES}

In many organisms, deficiency in condensin functions leads to a failure in anaphase chromosome segregation that is manifested by the formation of the so-called anaphase bridges (e.g., Hudson et al. 2003). The formation of anaphase bridges is likely to be a consequence of defects in resolving sister chromatids by metaphase. Consistent with this view, similar defective phenotypes are often observed in cells deficient in topoisomerase II. More recently, Chan et al. (2007) described a novel, hitherto unrecognized type of anaphase bridges, termed ultrafine DNA bridges (UFBs), that is observed even in normal cells (Chan et al. 2007). Unlike the conventional anaphase bridges, UFBs are not detectable with commonly used DNA dyes: they are instead recognized as "thread-like" structures decorated with antibodies against BLM (a RecQ helicase mutated in the Bloom's syndrome) and PICH (an SNF2-like helicase).

Subsequently, studies have shown that there are at least two different classes of UFBs (reviewed by Chan and Hickson 2011). The first class is derived primarily from centromeres whose resolution is delayed until anaphase onset (Fig. 5A). It is tempting to speculate that 


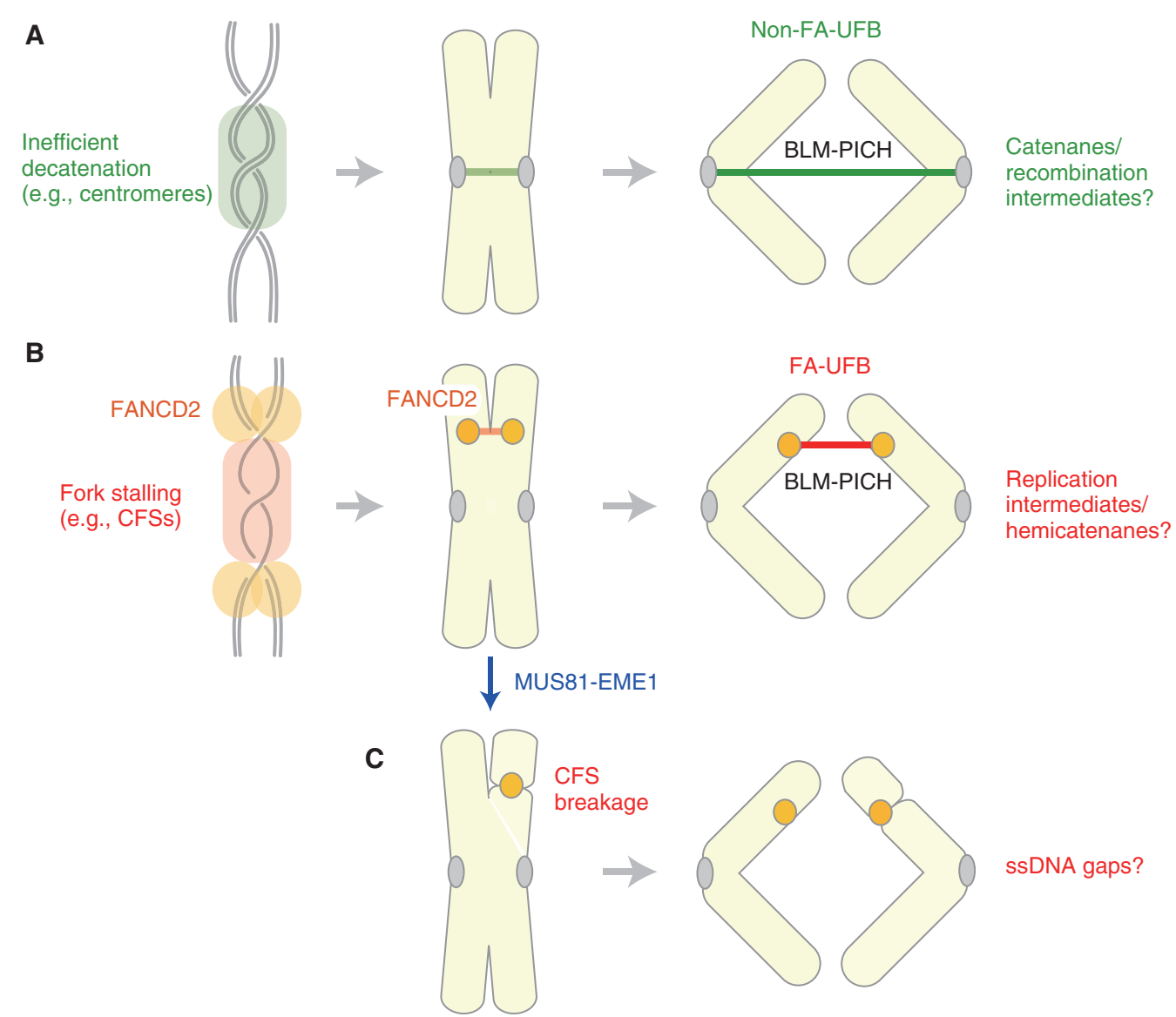

Figure 5. Segregation of difficult regions of chromosomes. Anaphase cells often display the so-called ultrafine DNA bridges (UFBs) that connect separating chromatids. They are almost invisible with conventional DNA dyes, yet can be visualized as threads positive for BLM (a RecQ helicase mutated in the Bloom's syndrome) and PICH (an SNF2-like helicase). (A) The first class of UFBs (non-Fanconi anemia (FA)-UFBs), primarily containing centromeric regions, is observed even under unperturbed conditions. It is hypothesized that inefficient decatenation (or incomplete processing of recombination intermediates) at centromeres generates such UFBs, which are subsequently resolved by the combined action of topoisomerase II and BLM. (B) The second class of UFBs is observed frequently under replication stress, especially at common fragile sites (CFSs). The unique feature of this class of UFBs (FA-UFBs) is that the FA protein FANCD2 localizes to the termini of BLM-PICHpositive bridges. The bridges could be resolved during anaphase as above, or cleaved and repaired in subsequent $\mathrm{G}_{1}$ phase. $(C)$ Alternatively, if the FACND2-positive replication intermediates are cleaved by the action of the MUS81-EME1 nuclease before metaphase, the resulting chromosomes display chromatid gaps or breaks characteristics of CFSs (i.e., CFS breakage). ssDNA, single-stranded DNA.

these "difficult-to-resolve" regions require an additional time and mechanism that could involve the BLM complex containing topoisomerase III. It is also important to note that centromeric regions are rich in repetitive sequences in animal cells, possibly involving a substantial level of recombination events even under un- perturbed conditions. Intriguingly, it has been reported that depletion of SMC2, a core subunit of condensins I and II, increases the frequency of UFBs (Lukas et al. 2011).

The second class of UFBs is often produced at common fragile sites (CFSs), chromosomal regions that are prone to form breaks or gaps 
during mitosis, in particular, following replication stress (reviewed by Debatisse et al. 2011; Ozeri-Galai et al. 2012). In this class of UFBs (Fig. 5B), Fanconi anemia (FA) proteins FANCD2 and FANCI are found at the termini (but not the bridge per se) of the bridges (Chan et al. 2009), implicating that they may be derived from replication intermediates created under replication stress. On the other hand, it has recently been shown that the MUS81-EME1 nuclease is responsible for chromatid breakages observed at CFSs (Fig. 5C) (Naim et al. 2013; Ying et al. 2013). Taken all together, mitotic cells could manage to separate replication intermediates through two alternative mechanisms: one is MUS81-mediated DNA cleavage, resulting in CFS breakage, and the other is BLM-mediated dissolution, involving UFB formation. It will be of great interest to determine whether condensins might contribute to either one or both of these processes and, if so, how. In a yeast model system for studying CFSs, CFS breakage has been shown to depend on condensin and topoisomerase II (Hashash et al. 2012). Conversely, an early study implicated the role of cohesin in suppressing CFS breakage in human cells ( $\mathrm{Mu}-$ sio et al. 2005).

\section{COORDINATING CHROMOSOME SEGREGATION WITH CYTOKINESIS}

Segregating chromosome arms in late anaphase must clear the path of the cleavage furrow before the onset of cytokinesis. If such temporal coordination were compromised, the cleavage furrow would "cut" lagging chromosome arms, resulting in segregation errors and DNA damage. To avoid these potential problems, eukaryotic cells have evolved a surveillance mechanism (known as the "NoCut pathway" in yeast and the "abscission checkpoint" in humans) that generates a signal to delay cytokinesis until chromosome segregation is complete (Norden et al. 2006; Steigemann et al. 2009). Aurora B, which localizes to the spindle midzone in anaphase, plays an important role in this surveillance mechanism both in yeast and humans, although their downstream effects apparently differ between the two organisms. A recent study in
C. elegans has shown that condensin I, but not condensin II, localizes to the spindle midzone in anaphase in an Aurora B-dependent manner (Bembenek et al. 2013). Loss of condensin I not only causes the formation of anaphase chromosome bridges, but also destabilizes the cleavage furrow, resulting in abnormal cytokinesis. Thus, condensin I appears to help generate a signal that delays cytokinesis. Consistent with this view, a subfraction of condensin has also been localized to the spindle midzone in fission yeast (Nakazawa et al. 2011). In retrospect, it is no mere coincidence that the original condensin mutants in fission yeast were isolated as those displaying the so-called "cut" phenotype, in which cytokinesis abnormally proceeds without proper segregation of chromosomes (Hirano et al. 1986). Elucidating the architectural and signaling network that coordinates chromosome segregation with cytokinesis will be an exciting topic in the field of mitosis.

\section{CONCLUDING REMARKS}

In this article, I have briefly summarized recent progress in the field of mitotic chromosome dynamics. Since the discoveries of SMC proteins, cohesin, and condensins, almost 20 years ago, the field has gained a fundamental body of knowledge as to how chromosomes per se change their own conformations to support their own segregation. Although many, if not all, molecular players have been identified and the overall framework has been constructed, important and immense challenges lie ahead of us. First, we still have very limited information concerning the exact mechanisms of action of cohesin and condensins. Full reconstitution of these SMC complexes (along with their regulatory factors) from recombinant subunits, combined with development of robust functional assays, will be a daunting, yet inevitable, task to push the field forward (Murayama and Uhlmann 2014). Gaining more quantitative information regarding the dynamics and actions of these chromosomal components both in vivo and in vitro will also be required before we are able to initiate practical efforts toward mathematical modeling of chromosome segregation. 
Second, it has become increasingly clear that the machineries essential for mitotic chromosome dynamics are also directly involved in a number of nonmitotic chromosomal functions, such as gene expression, recombination/repair, and formation/maintenance of interphase chromosome architecture. The recent appreciation that hypomorphic mutations or misregulation of essential chromosomal components potentially cause severe developmental defects in humans is an exciting, conceptual progress that has prompted researchers in different fields to talk to each other. Such trends will undoubtedly continue in the years to come. It will also be important to determine to what extent mutations or misregulation of cohesin and condensin subunits might contribute to human cancers (Ham et al. 2007; Balbás-Martínez et al. 2013; Guo et al. 2013; Kon et al. 2013; Solomon et al. 2013). Third, and, finally, all issues described above need to be addressed, also, from an evolutionary point of view. To what extent might eukaryotes and prokaryotes share the basic mechanisms of chromosome segregation? How could the conserved set of protein components manage to organize eukaryotic chromosomes in a wide variety of organisms whose average chromosome lengths range from $\sim 1 \mathrm{Mb}$ (budding yeast) to $\sim 1800 \mathrm{Mb}$ (newt)? Attempts to answer these questions will help lead us to a deep understanding of the origin of life, as well as the biodiversity we see currently on earth.

\section{ACKNOWLEDGMENTS}

I thank members of the Hirano Laboratory and colleagues in the field for stimulating discussions. The work of the author's laboratory is supported by a Grant-in-Aid for Specially Promoted Research.

\section{REFERENCES}

Abe S, Nagasaka K, Hirayama Y, Kozuka-Hata H, Oyama M, Aoyagi Y, Obuse C, Hirota T. 2011. The initial phase of chromosome condensation requires Cdk1-mediated phosphorylation of the CAP-D3 subunit of condensin II. Genes Dev 25: 863-874.

Bachellier-Bassi S, Gadal O, Bourout G, Nehrbass U. 2008. Cell cycle-dependent kinetochore localization of con- densin complex in Saccharomyces cerevisiae. J Struct Biol 162: 248-259.

Balbás-Martínez C, Sagrera A, Carrillo-de-Santa-Pau E, Earl J, Márquez M, Vazquez M, Lapi E, Castro-Giner F, Beltran S, Bayés M, et al. 2013. Recurrent inactivation of STAG2 in bladder cancer is not associated with aneuploidy. Nat Genet 45: 1464-1469.

Bauer CR, Hartl TA, Bosco G. 2012. Condensin II promotes the formation of chromosome territories by inducing axial compaction of polyploid interphase chromosomes. PLoS Genet 8: e1002873.

Beckouët F, Hu B, Roig MB, Sutani T, Komata M, Uluocak P, Katis VL, Shirahige K, Nasmyth K. 2010. An Smc3 acetylation cycle is essential for establishment of sister chromatid cohesion. Mol Cell 39: 689-699.

Bellows AM, Kenna MA, Cassimeris L, Skibbens RV. 2003. Human EFO1p exhibits acetyltransferase activity and is a unique combination of linker histone and Ctf7p/Ecolp chromatin cohesion establishment domains. Nucl Acids Res 31: 6334-6343.

Bembenek JN, Verbrugghe KJC, Khanikar J, Csankovszki G, Chan RC. 2013. Condensin and the spindle midzone prevent cytokinesis failure induced by chromatin bridges in C. elegans embryos. Curr Biol 23: 937-946.

Ben-Shahar TR, Heeger S, Lehane C, East P, Flynn H, Skehel M, Uhlmann F. 2008. Ecol-dependent cohesin acetylation during establishment of sister chromatid cohesion. Science 321: 563-566.

Bisht KK, Daniloski Z, Smith S. 2013. SA1 binds directly to DNAvia its unique AT-hook to promote sister chromatid cohesion at telomeres. J Cell Sci 126: 3493-3503.

Borges V, Lehane C, Lopez-Serra L, Flynn H, Skehel M, Rolef Ben-Shahar T, Uhlmann F. 2010. Hos1 deacetylates Smc3 to close the cohesin acetylation cycle. Mol Cell 39: 677688.

Buster DW, Daniel SG, Nguyen HQ, Windler SL, Skwarek LC, Peterson M, Roberts M, Meserve JH, Hartl T, Klebba JE, et al. 2013. SCFSlimb ubiquitin ligase suppresses condensin II-mediated nuclear reorganization by degrading Cap-H2. J Cell Biol 201: 49-63.

Canudas S, Smith S. 2009. Differential regulation of telomere and centromere cohesion by the Scc3 homologues SA1 and SA2, respectively, in human cells. J Cell Biol 187: $165-173$.

Chan KL, Hickson ID. 2011. New insights into the formation and resolution of ultra-fine anaphase bridges. Semin Cell Dev Biol 22: 906-912.

Chan K-L, North PS, Hickson ID. 2007. BLM is required for faithful chromosome segregation and its localization defines a class of ultrafine anaphase bridges. $E M B O J$ 26: 3397-3409.

Chan KL, Palmai-Pallag T, Ying S, Hickson ID. 2009. Replication stress induces sister-chromatid bridging at fragile site loci in mitosis. Nat Cell Biol 11: 753-760.

Chatterjee A, Zakian S, Hu X-W, Singleton MR. 2013. Structural insights into the regulation of cohesion establishment by Wpll. EMBO J 32: 677-687.

Collette KS, Petty EL, Golenberg N, Bembenek JN, Csankovszki G. 2011. Different roles for Aurora B in condensin targeting during mitosis and meiosis. J Cell Sci 124: 3684-3694. 
T. Hirano

Deardorff MA, Bando M, Nakato R, Watrin E, Itoh T, Minamino M, Saitoh K, Komata M, Katou Y, Clark D, et al. 2012. HDAC8 mutations in Cornelia de Lange syndrome affect the cohesin acetylation cycle. Nature 489: 313-317.

Debatisse M, Le Tallec B, Letessier A, Dutrillaux B, Brison O. 2011. Common fragile sites: Mechanisms of instability revisited. Trends Genet 28: 22-32.

Fujiwara T, Tanaka K, Kuroiwa T, Hirano T. 2013. Spatiotemporal dynamics of condensins I and II: Evolutionary insights from the primitive red alga Cyanidioschyzon merolae. Mol Biol Cell 24: 2515-2527.

Gandhi R, Gillespie PJ, Hirano T. 2006. Wapl is a cohesinbinding protein that promotes sister chromatid resolution in mitotic prophase. Curr Biol 16: 2406-2417.

Gause M, Misulovin Z, Bilyeu A, Dorsett D. 2010. Dosagesensitive regulation of cohesin chromosome binding and dynamics by Nipped-B, Pds5, and Wapl. Mol Cell Biol 30: 4940-4951.

Gerlich D, Hirota T, Koch B, Peters J-M, Ellenberg J. 2006a. Condensin I stabilizes chromosomes mechanically through a dynamic interaction in living cells. Curr Biol 16: $333-344$.

Gerlich D, Koch B, Dupeux F, Peters J-M, Ellenberg J. 2006b. Live-cell imaging reveals a stable cohesin-chromatin interaction after but not before DNA replication. Curr Biol 16: $1571-1578$.

Goshima G, Wollman R, Goodwin SS, Zhang N, Scholey JM, Vale RD, Stuurman N. 2007. Genes required for mitotic spindle assembly in Drosophila S2 cells. Science 316: 417 421.

Green LC, Kalitsis P, Chang TM, Cipetic M, Kim JH, Marshall O, Turnbull L, Whitchurch CB, Vagnarelli P, Samejima K, et al. 2012. Contrasting roles of condensin I and II in mitotic chromosome formation. J Cell Sci 125: 15911604.

Gruber R, Zhou Z, Sukchev M, Joerss T, Frappart P-O, Wang Z-Q. 2011. MCPH1 regulates the neuroprogenitor division mode by coupling the centrosomal cycle with mitotic entry through the Chk1-Cdc25 pathway. Nat Cell Biol 13: 1325-1334.

Guacci V, Koshland D, Strunnikov A. 1997. A direct link between sister chromatid cohesion and chromosome condensation revealed through the analysis of MCD1 in S. cerevisiae. Cell 91: 47-57.

Guo G, Sun X, Chen C, Wu S, Huang P, Li Z, Dean M, Huang Y, Jia W, Zhou Q, et al. 2013. Whole-genome and wholeexome sequencing of bladder cancer identifies frequent alterations in genes involved in sister chromatid cohesion and segregation. Nat Genet 45: 1459-1463.

Ham MF, Takakuwa T, Rahadiani N, Tresnasari K, Nakajima H, Aozasa K. 2007. Condensin mutations and abnormal chromosomal structures in pyothorax-assocaited lymphoma. Cancer Sci 98: 1041-1047.

Harrison B, Hoang M, Bloom K. 2009. Persistent mechanical linkage between sister chromatids throughout anaphase. Chromosoma 118: 633-645.

Hartl TA, Smith HF, Bosco G. 2008. Chromosome alignment and transvection are antagonized by condensin II. Science 322: 1384-1387.

Hashash N, Johnson AL, Cha RS. 2012. Topoisomerase II- and condensin-dependent breakage of MEC1(ATR)- sensitive fragile sites occurs independently of spindle tension, anaphase, or cytokinesis. PLoS Genet 8: e1002978.

Heidinger-Pauli JM, Mert O, Davenport C, Guacci V, Koshland D. 2010. Systematic reduction of cohesin differentially affects chromosome segregation, condensation, and DNA repair. Curr Biol 20: 957-963.

Herzog S, Nagarkar Jaiswal S, Urban E, Riemer A, Fischer S, Heidmann SK. 2013. Functional dissection of the Drosophila melanogaster condensin subunit Cap-G reveals its exclusive association with condensin I. PLoS Genet 9: e1003463.

Hirano T. 2012. Condensins: Universal organizers of chromosomes with diverse functions. Genes Dev 26: 16591678.

Hirano T, Funahashi S, Uemura T, Yanagida M. 1986. Isolation and characterization of Schizosaccharomyces pombe cut mutants that block nuclear division but not cytokinesis. EMBO J 5: 2973-2979.

Hirano T, Kobayashi R, Hirano M. 1997. Condensins, chromosome condensation protein complexes containing XCAP-C, XCAP-E and a Xenopus homolog of the Drosophila Barren protein. Cell 89: 511-521.

Hirota T, Gerlich D, Koch B, Ellenberg J, Peters JM. 2004. Distinct functions of condensin I and II in mitotic chromosome assembly. J Cell Sci 117: 6435-6445.

Hudson DF, Vagnarelli P, Gassmann R, Earnshaw WC. 2003. Condensin is required for nonhistone protein assembly and structural integrity of vertebrate chromosomes. Dev Cell 5: 323-336.

Ivanov D, Schleiffer A, Eisenhaber F, Mechtler K, Haering CH, Nasmyth K. 2002. Ecol is a novel acetyltransferase that can acetylate proteins involved in cohesion. Curr Biol 12: $323-328$.

Joyce EF, Williams BR, Xie T, Wu C-T. 2012. Identification of genes that promote or antagonize somatic homolog pairing using a high-throughput FISH-based screen. PLoS Genet 8: e1002667.

Kagami Y, Nihira K, Wada S, Ono M, Honda M, Yoshida K. 2014. Mps1 phosphorylation of condensin II controls chromosome condensation at the onset of mitosis. $J$ Cell Biol 205: 781-790.

Kimura K, Hirano M, Kobayashi R, Hirano T. 1998. Phosphorylation and activation of $13 \mathrm{~S}$ condensin by Cdc2 in vitro. Science 282: 487-490.

Kireeva N, Lakonishok M, Kireev I, Hirano T, Belmont AS. 2004. Visualization of early chromosome condensation: A hierarchical folding, axial glue model of chromosome structure. J Cell Biol 166: 775-785.

Kitajima T, Sakuno T, Ishiguro K-I, Iemura S-I, Natsume T, Kawashima SA, Watanabe Y. 2006. Shugoshin collaborates with protein phosphatase $2 \mathrm{~A}$ to protect cohesin. Nature 441: 46-52.

Kon A, Shih L-Y, Minamino M, Sanada M, Shiraishi Y, Nagata Y, Yoshida K, Okuno Y, Bando M, Nakato R, et al. 2013. Recurrent mutations in multiple components of the cohesin complex in myeloid neoplasms. Nat Genet 45: $1232-1237$.

Kueng S, Hegemann B, Peters BH, Lipp JJ, Schleiffer A, Mechtler K, Peters J-M. 2006. Wapl controls the dynamic association of cohesin with chromatin. Cell 127: 955967. 
Lafont AL, Song J, Rankin S. 2010. Sororin cooperates with the acetyltransferase Eco2 to ensure DNA replication-dependent sister chromatid cohesion. Proc Natl Acad Sci 107: 20364-20369.

Lee J, Ogushi S, Saitou M, Hirano T. 2011. Condensins I and II are essential for construction of bivalent chromosomes in mouse oocytes. Mol Biol Cell 22: 3465-3477.

Lipp JJ, Hirota T, Poser I, Peters J-M. 2007. Aurora B controls the association of condensin I but not condensin II with mitotic chromosomes. J Cell Sci 120: 1245-1255.

Losada A, Hirano M, Hirano T. 1998. Identification of Xenopus SMC protein complexes required for sister chromatid cohesion. Genes Dev 12: 1986-1997.

Losada A, Yokochi T, Kobayashi R, Hirano T. 2000. Identification and characterization of SA/Scc3p subunits in the Xenopus and human cohesin complexes. J Cell Biol 150: 405-416.

Losada A, Hirano M, Hirano T. 2002. Cohesin release is required for sister chromatid resolution, but not for condensin-mediated compaction, at the onset of mitosis. Genes Dev 16: 3004-3016.

Lukas C, Savic V, Bekker-Jensen S, Doil C, Neumann B, Pedersen RS, Grøfte M, Chan KL, Hickson ID, Bartek J, et al. 2011. 53BP1 nuclear bodies form around DNA lesions generated by mitotic transmission of chromosomes under replication stress. Nat Cell Biol 13:243-253.

Marko JF. 2008. Micromechanical studies of mitotic chromosomes. Chromosome Res 16: 469-497.

Mazumdar M, Sundareshan S, Misteli T. 2004. Human chromokinesin KIF4A functions in chromosome condensation and segregation. J Cell Biol 166: 613-620.

Michaelis C, Ciosk R, Nasmyth K. 1997. Cohesins: Chromosomal proteins that prevent premature separation of sister chromatids. Cell 91: 35-45.

Murayama Y, Uhlmann F. 2014. Biochemical reconstitution of topological DNA binding by the cohesin ring. Nature 505: $367-371$.

Musio A, Montagna C, Mariani T, Tilenni M, Focarelli ML, Brait L, Indino E, Benedetti PA, Chessa L, Albertini A, et al. 2005. SMC1 involvement in fragile site expression. Hum Mol Genet 14: 525-533.

Naim V, Wilhelm T, Debatisse M, Rosselli F. 2013. ERCC1 and MUS81-EME1 promote sister chromatid separation by processing late replication intermediates at common fragile sites during mitosis. Nat Cell Biol 15: 1008-1015.

Nakajima M, Kumada K, Hatakeyama K, Noda T, Peters J-M, Hirota T. 2007. The complete removal of cohesin from chromosome arms depends on separase. J Cell Sci 120: $4188-4196$.

Nakazawa N, Mehrotra R, Ebe M, Yanagida M. 2011. Condensin phosphorylated by the Aurora-B-like kinase Ark1 is continuously required until telophase in a mode distinct from Top2. J Cell Sci 124: 1795-1807.

Nasmyth K. 2011. Cohesin: A catenase with separate entry and exit gates? Nat Cell Biol 13: 1170-1177.

Nishiyama T, Ladurner R, Schmitz J, Kreidl E, Schleiffer A, Bhaskara V, Bando M, Shirahige K, Hyman AA, Mechtler $\mathrm{K}$, et al. 2010. Sororin mediates sister chromatid cohesion by antagonizing Wapl. Cell 143: 737-749.

Norden C, Mendoza M, Dobbelaere J, Kotwaliwale CV, Biggins S, Barral Y. 2006. The NoCut pathway links comple- tion of cytokinesis to spindle midzone function to prevent chromosome breakage. Cell 125: 85-98.

Ono T, Losada A, Hirano M, Myers MP, Neuwald AF, Hirano T. 2003. Differential contributions of condensin I and condensin II to mitotic chromosome architecture in vertebrate cells. Cell 115: 109-121.

Ono T, Fang Y, Spector D, Hirano T. 2004. Spatial and temporal regulation of condensins I and II in mitotic chromosome assembly in human cells. Mol Biol Cell 15: 3296-3308.

Ono T, Yamashita D, Hirano T. 2013. Condensin II initiates sister chromatid resolution during S phase. J Cell Biol 200: 429-441.

Ouyang Z, Zheng G, Song J, Borek DM, Otwinowski Z, Brautigam CA, Tomchick DR, Rankin S, Yu H. 2013. Structure of the human cohesin inhibitor Wapl. Proc Natl Acad Sci 110: 11355-11360.

Ozeri-Galai E, Bester AC, Kerem B. 2012. The complex basis underlying common fragile site instability in cancer. Trends Genet 28: 295-302.

Peters J-M, Tedeschi A, Schmitz J. 2008. The cohesin complex and its roles in chromosome biology. Genes Dev 22: 3089-3114.

Petrova B, Dehler S, Kruitwagen T, Hériché J-K, Miura K, Haering CH. 2012. Quantitative analysis of chromosome condensation in fission yeast. Mol Cell Biol 33: 984-998.

Remeseiro S, Cuadrado A, Carretero M, Martínez P, Drosopoulos WC, Cañamero $\mathrm{M}$, Schildkraut CL, Blasco MA, Losada A. 2012a. Cohesin-SAl deficiency drives aneuploidy and tumourigenesis in mice due to impaired replication of telomeres. EMBO J 31: 2076-2089.

Remeseiro S, Cuadrado A, Gómez-López G, Pisano DG, Losada A. 2012b. A unique role of cohesin-SA1 in gene regulation and development. EMBO J 31: 2090-2102.

Renshaw MJ, Ward JJ, Kanemaki M, Natsume K, Nédélec FJ, Tanaka TU. 2010. Condensins promote chromosome recoiling during early anaphase to complete sister chromatid separation. Dev Cell 19: 232-244.

Sakamoto T, Inui YT, Uraguchi S, Yoshizumi T, Matsunaga S, Mastui M, Umeda M, Fukui K, Fujiwara T. 2011. Condensin II alleviates DNA damage and is essential for tolerance of boron overload stress in Arabidopsis. Plant Cell 23: 3533-3546.

Samejima I, Matsumoto T, Nakaseko Y, Beach D, Yanagida M. 1993. Identification of seven new cut genes involved in Schizosaccharomyces pombe mitosis. J Cell Sci 105: 135143.

Samejima K, Samejima I, Vagnarelli P, Ogawa H, Vargiu G, Kelly DA, de Lima Alves F, Kerr A, Green LC, Hudson DF, et al. 2012. Mitotic chromosomes are compacted laterally by KIF 4 and condensin and axially by topoisomerase II $\alpha$. J Cell Biol 199: 755-770.

Savvidou E, Cobbe N, Steffensen S, Cotterill S, Heck MMS. 2005. Drosophila CAP-D2 is required for condensin complex stability and resolution of sister chromatids. J Cell Sci 118: $2529-2543$.

Shindo N, Kumada K, Hirota T. 2012. Separase sensor reveals dual roles for separase coordinating cohesin cleavage and cdk1 inhibition. Dev Cell 23: 112-123. 
T. Hirano

Shintomi K, Hirano T. 2009. Releasing cohesin from chromosome arms in early mitosis: Opposing actions of Wapl-Pds5 and Sgo1. Genes Dev 23: 2224-2236.

Shintomi K, Hirano T. 2010. Sister chromatid resolution: A cohesin releasing network and beyond. Chromosoma 119: 459-467.

Shintomi K, Hirano T. 2011. The relative ratio of condensin I to II determines chromosome shapes. Genes Dev 25: 1464-1469.

Solomon DA, Kim T, Diaz-Martinez LA, Fair J, Elkahloun AG, Harris BT, Toretsky JA, Rosenberg SA, Shukla N, Ladanyi M, et al. 2011. Mutational inactivation of STAG2 causes aneuploidy in human cancer. Science 333: $1039-1043$.

Solomon DA, Kim J-S, Bondaruk J, Shariat SF, Wang Z-F, Elkahloun AG, Ozawa T, Gerard J, Zhuang D, Zhang S, et al. 2013. Frequent truncating mutations of STAG2 in bladder cancer. Nat Genet 45: 1428-1430.

Steigemann P, Wurzenberger C, Schmitz MHA, Held M, Guizetti J, Maar S, Gerlich DW. 2009. Aurora B-mediated abscission checkpoint protects against tetraploidization. Cell 136: 473-484.

St-Pierre J, Douziech M, Bazile F, Pascariu M, Bonneil E, Sauvé V, Ratsima H, D’Amours D. 2009. Polo kinase regulates mitotic chromosome condensation by hyperactivation of condensin DNA supercoiling activity. Mol Cell 34: $416-426$

Sumara I, Vorlaufer E, Stukenberg PT, Kelm O, Redermann N, Nigg EA, Peters J-M. 2002. The dissociation of cohesin from chromosomes in prophase is regulated by Polo-like kinase. Mol Cell 9: 515-525.

Sutani T, Yuasa T, Tomonaga T, Dohmae N, Takio K, Yanagida M. 1999. Fission yeast condensin complex: Essential roles of non-SMC subunits for condensation and cdc2 phosphorylation of Cut3/SMC4. Genes Dev 13: 22712283.

Tada K, Susumu H, Sakuno T, Watanabe Y. 2011. Condensin association with histone H2A shapes mitotic chromosomes. Nature 474: 477-483.
Takahashi Y, Dulev S, Liu X, Hiller NJ, Zhao X, Strunnikov A. 2008. Cooperation of sumoylated chromosomal proteins in rDNA maintenance. PLoS Genet 4: e1000215.

Thornton GK, Woods CG. 2009. Primary microcephaly: Do all roads lead to Rome? Trends Genet 25: 501-510.

Trimborn M, Schindler D, Neitzel H, Hirano T. 2006. Misregulated chromosome condensation in $\mathrm{MCPH} 1$ primary microcephaly is mediated by condensin II. Cell Cycle 5: 322-326.

Uhlmann F, Lottspeich F, Nasmyth K. 1999. Sister-chromatid separation at anaphase onset is promoted by cleavage of the cohesin subunit Sccl. Nature 400: 37-42.

Unal E, Heidinger-Pauli JM, Kim W, Guacci V, Onn I, Gygi SP, Koshland DE. 2008. A molecular determinant for the establishment of sister chromatid cohesion. Science 321: 566-569.

Vega H, Waisfisz Q, Gordillo M, Sakai N, Yanagihara I, Yamaga M, van Gosliga D, Kayserili H, Xu C, Ozono K, et al. 2005. Roberts syndrome is caused by mutations in ESCO2, a human homolog of yeast ECO1 that is essential for the establishment of sister chromatid cohesion. Nat Genet 37: 468-470.

Xiong B, Lu S, Gerton JL. 2010. Hos 1 is a lysine deacetylase for the Smc3 subunit of cohesin. Curr Biol 20: 16601665.

Yamashita D, Shintomi K, Ono T, Gavvovidis I, Schindler D, Neitzel H, Trimborn M, Hirano T. 2011. MCPH1 regulates chromosome condensation and shaping as a composite modulator of condensin II. J Cell Biol 194: $841-$ 854.

Ying S, Minocherhomji S, Chan K-L, Palmai-Pallag T, Chu WK, Wass T, Mankouri HW, Liu Y, Hickson ID. 2013. MUS81 promotes common fragile site expression. Nat Cell Biol 15: 1001-1007.

Zhang J, Shi X, Li Y, Kim B-J, Jia J, Huang Z, Yang T, Fu X, Jung SY, Wang Y, et al. 2008. Acetylation of Smc3 by Ecol is required for s phase sister chromatid cohesion in both human and yeast. Mol Cell 31: 143-151. 


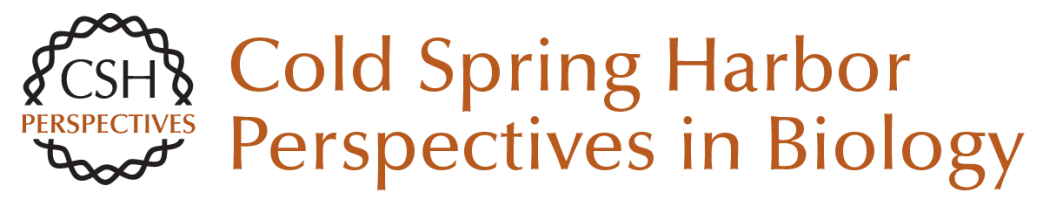

\section{Chromosome Dynamics during Mitosis}

Tatsuya Hirano

Cold Spring Harb Perspect Biol 2015; doi: 10.1101/cshperspect.a015792 originally published online February 26, 2015

\section{Subject Collection Mitosis}

Emergent Properties of the Metaphase Spindle Simone Reber and Anthony A. Hyman

Meiosis: An Overview of Key Differences from Mitosis

Hiroyuki Ohkura

Cytokinesis in Animal Cells

Pier Paolo D'Avino, Maria Grazia Giansanti and Mark Petronczki

The Centrosome and Its Duplication Cycle Jingyan Fu, Iain M. Hagan and David M. Glover

The Role of Model Organisms in the History of Mitosis Research

Mitsuhiro Yanagida
Chromosome Dynamics during Mitosis

Tatsuya Hirano

The Centromere: Epigenetic Control of

Chromosome Segregation during Mitosis

Frederick G. Westhorpe and Aaron F. Straight

The Biochemistry of Mitosis

Samuel Wieser and Jonathon Pines

Aurea Mediocritas: The Importance of a Balanced

Genome

Gianluca Varetti, David Pellman and David J.

Gordon

The Kinetochore

Iain M. Cheeseman

For additional articles in this collection, see http://cshperspectives.cshlp.org/cgi/collection/

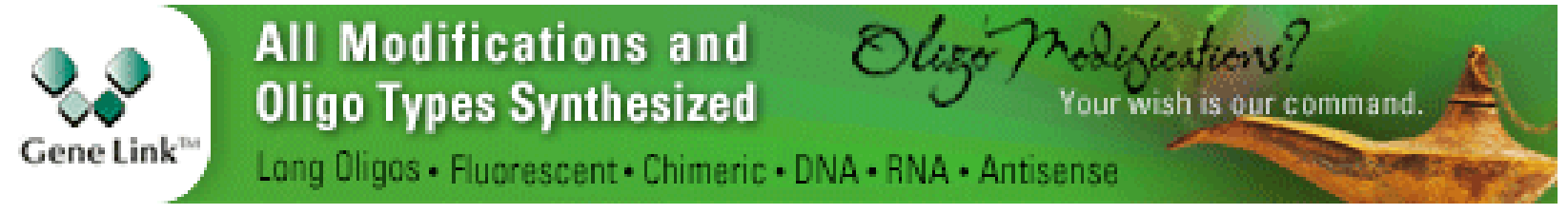

Copyright @ 2015 Cold Spring Harbor Laboratory Press; all rights reserved 